\title{
Expectations, Intentions, and Actions in Human-Robot Interaction
}

\author{
Marc Hanheide • Manja Lohse • Hendrik Zender
}

Published online: 1 February 2012

(C) Springer Science \& Business Media BV 2012

Human-robot interaction is becoming increasingly complex through the growing number of abilities, both cognitive and physical, available to today's robots. At the same time, interaction is still often difficult because the users do not understand the robots' internal states, expectations, intentions, and actions. Vice versa, robots lack understanding of the users' expectations, intentions, actions, and social signals.

Many studies have been conducted unveiling the importance of properly designed adaptive human-robot interaction strategies in general and appropriate feedback in particular. Robotic systems have been built that reflect the progress in the different fields in robotics with regard to learning, autonomous behaviours, safe navigation, and manipulation. However, integrated approaches to

- understanding the user and her expectations, intentions, and actions,

- transparently communicating to the user what the robot understood or expected,

This work has been supported by the European Commission under contract number FP7-ICT-215181-CogX.

M. Hanheide ( $\varangle)$

School of Computer Science, University of Lincoln, Lincoln, United Kingdom

e-mail: mhanheide@lincoln.ac.uk

M. Lohse

Research Institute for Cognition and Robotics (CoR-Lab), Applied Informatics, Bielefeld University, Bielefeld, Germany e-mail: mlohse@techfak.uni-bielefeld.de

H. Zender

Language Technology Lab, German Research Center for Artificial Intelligence (DFKI), Saarbrücken, Germany e-mail: zender@dfki.de
- and designing appropriate robot behaviours based on its understanding of the world

are still in their infancy.

This special issue bundles recent advances in addressing these challenges. The key questions are how meeting or failing the user's expectations influences the efficiency and effectiveness of human-robot interaction; how more effective and efficient interaction with humans can be achieved using modalities available to a robot; how robots can be equipped with models enabling them to understand their users' state of mind; and similarly, how they can make their own expectations and states explicit through eligible communication channels.

Each of the seven articles in this issue highlights different aspects around the central theme of expectations, intentions, and actions in human-robot interaction. The topics covered range from recognition of verbal and non-verbal cues of intentions and expectations, to verbalisation and presentation techniques that make internal processing of the robot accessible to the human. In the following we give a brief overview of the contents of the articles.

Komatsu, Kurosowa, and Yamada ask "How does the Difference between Users' Expectations and Perceptions about a Robotic Agent Affect Their Behavior?" They call this difference the adaption gap and research how it affects the decisions of the users in a game. Their findings show that the participants with positive adaptation gap signs (i.e., the robot performed better than they expected) accepted the robot's suggestions more often than users with a negative adaptation gap sign (i.e., the robot performed worse than they expected). This work shows that expectations indeed play a major role in human-robot interaction.

The paper by Rosenthal, Veloso, and Dey deals with the problem of "Acquiring Accurate Human Responses to 
Robots' Questions.” They identify and evaluate four different types of state information that a robot can use to ground non-supervisors in its state when requesting help. The authors describe what they believe to be the most appropriate combination of information to communicate the robot's intentions and to trigger the right actions by the users.

Two papers in this issue focus on the analysis of human behaviour to facilitate contingency in interaction with a robot. Contingency is inherently related to expectations in interaction, as contingency can only be achieved if the mutual expectations of the interactants are met. However, the objectives of this analysis of contingency in the two articles differ: While Lohan et al. propose a model to detect tutoring turns in an interactive learning setting informed by adult-infant interaction, Lee et al. investigate the potential of multimodal cues to help turn-taking in natural interaction.

Focusing on the perception of intention and emotions, Yohanan and MacLean define different categories of intent in the interaction with an affective artificial creature. Those intents are related to emotional states that are haptically conveyed to a robot. The contribution proposes patterns of gesture use for emotional expression as well as a touch dictionary used to convey those different intents.

The paper by Lemaignan et al. entitled "Grounding the Interaction: Anchoring Situated Discourse in Everyday Human-Robot Interaction" presents a robotic system that can engage in situated dialogues with a human about shared tabletop environments. The system comprises perceptual modalities that allow it to build symbolic representations of the environment. One of the key aspects of the approach is that the system is capable of perspective taking - effectively allowing the robot to take into account the human's perspective as well as its own when interpreting and generating utterances.
Finally, Salem et al. present work on the generation of behaviour by the robot to facilitate the understanding of robot's intentions and expectations. They propose a novel gesture generation mechanism evaluated on a humanoid platform to produce co-verbal hand and arm gestures.

Some of these papers are extended versions of work presented in two preceding workshops, others have been submitted to an open call for papers. This special issue appears as a follow-up to the workshops on Interactive Communication for Autonomous Intelligent Robots (ICAIR), held at the ICRA 2010 conference in Anchorage, AK, USA, and on the Role of Expectations in Intuitive Human-Robot Interaction, held at the HRI 2011 conference in Lausanne, Switzerland.

Therefore, we wish to express our gratitude to our colleagues who helped us organise these workshops as well as this special issue. Many thanks go to the co-organisers of our workshops: Verena Hafner (Humboldt University Berlin), Geert-Jan M. Kruijff (DFKI Saarbrücken), Joachim Meyer (Ben-Gurion University of the Negev), Yukie Nagai (Osaka University), and Britta Wrede (Bielefeld University). Special thanks go to Professor Shuzhi Sam Ge, who encouraged us to prepare this special issue based on the workshop outcomes, and to the reviewers for their support in maintaining the high standard of this issue: Kai Oliver Arras, Cindy L. Bethel, Laura Boccanfuso, Heriberto Cuayáhuitl, Frank Hegel, Thomas Holz, David O. Johnson, Takayuki Kanda, Alexandra Kirsch, Nate Koenig, Min Kyung Lee, Ross Mead, Yukie Nagai, Hirotaka Osawa, Julia Peltason, Annika Peters, Karola Pitsch, Astrid Marieke von der Pütten, Bogdan Raducanu, Subramanian Ramamoorthy, Laurel Riek, Pericle Salvini, Sven R. Schmidt-Rohr, Elin Anna Topp, Alan Richard Wagner, Yueh-Hsuan Weng, and Britta Wrede. 